\title{
APPLICATION OF JUDD-OFELT THEORY UPON CHLOFLUOROPHOSPHATE GLASS DOPED WITH ERBIUM
}

\author{
A. Babai", K. E. Aiadi, O. Bentouila, F. Rehoma and M. Poulain \\ Équipe Optoélectronique, Laboratoire LENREZA, Université Kasdi Merbah- \\ Ouargla, 30000 Ouargla, Algérie
}

Received: 25 November 2012 / Accepted: 02 December 2012 / Published online: 30 June 2012

\begin{abstract}
A series of erbium doped glasses chlorofluorophosphates were prepared and characterized. The absorption spectra were analyzed to determine the Judd-Ofelt parameters. The optical performance of these doped glasses suggesting the relevance of these glasses for optical fiber/ wave guide lasers and optical amplifiers.
\end{abstract}

Keys words: glass doped, rare earth, erbium, Judd-Ofelt parameters, laser, and optical amplifier.

\section{INTRODUCTION}

L'utilisation des verres en optique active fait l'objet des recherches durant les dernières décennies. Les verres deviennent actifs lorsqu'ils sont dopés par de faibles quantités d'ions luminescents telles que les ions de terres-rares (TR). Les terres rares sont utilisées comme dopants en raison de leurs nombreuses transitions dans le visible et l'infrarouge et de leur relative insensibilité à la matrice dans laquelle sont introduites. Les verres dopés terres rares sont utilisés comme des composants intégrés actifs en télécommunication: les lasers et les amplificateurs optiques. L'étude spectroscopique de ces verres fournit des informations sur des différents paramètres (probabilité de transition, durée de vie, section efficace d'absorption, section efficace d'émission...) qui sont importants dans la réalisation de ces composantes actifs.

Author Correspondence, e-mail: afaf.babai@gmail.com

ICID: 1020735 
Parmi les verres dopés terres rares, les verres fluorophosphates montrent des avantages importants. Ces verres sont caractérisés par une structure de réseau compliquée, teneur élevée des ions terres rares et une large bande de gain. Ils sont aussi possèdent une basse énergie de phonons, une transmittance allant de l'ultraviolet à l'infrarouge et un faible indice de réfraction non-linéaire [1-2-3]. En raison de ces caractéristiques, les verres fluorophosphate dopés avec différents ions de terre rare: $\mathrm{Nd}^{3+}, \mathrm{Er}^{3+}, \mathrm{Yb}^{3+}, \mathrm{Ho}^{3+}$, etc., sont largement répandus dans la technologie laser et amplificateurs optiques.

Dans ce travail, Nous avons étudié en particulier un verre de Chloro-fluorophosphate dopé avec $\mathrm{Er}^{+3}$.

\section{PROCEDURE EXPERIMENTALE}

\subsection{Synthèse des verres}

Le verre fluorophosphate, dont la composition: NaPO3- $\mathrm{PbCl} 2-\mathrm{ErF} 3$ avec la concentration en erbium égale à $0,722 \times 10^{20}$ ions $/ \mathrm{cm}^{3}$, a été synthétisé dans le laboratoire des matériaux photoniques Université de Rennes 1 (France). Les produits de départ utilisés sont rassemblés dans le tableau. 1:

Tableau 1. Masse molaire des produits de départ

\begin{tabular}{|c|c|c|}
\hline Produit & Masse molaire $\left(\mathrm{g} / \mathrm{cm}^{3}\right)$ & Facteur molaire (\%100) \\
\hline $\mathrm{NaPO}_{3}$ & 101,96 & 90 \\
\hline $\mathrm{PbCl}_{2}$ & 278,09 & 9 \\
\hline $\mathrm{ErF}_{3}$ & 224,25 & 1 \\
\hline
\end{tabular}

\subsection{Analyse thermiques}

La température de la transition vitreuse de notre verre à été mesurées par Calorimétrie Différentielle à Balayage (appelée encore: Analyse Calorimétrique Différentielle, en Anglais: Differential Scanning Calorimetry): D. S. C. la valeur de Tg a été trouvée environ de $250^{\circ} \mathrm{C}$.

\subsection{Mesures de densité}

La densité expérimentale $d_{\exp }$ des échantillons de verre a été mesurée en utilisant la méthode du pycnomètre à Hélium. L'appareillage utilisé est un ACCUPYC 1330 (MICROMERITICS à déplacement d'hélium (He). Chaque mesure a été effectuée 5 
fois, puis est moyennée afin d'obtenir des résultats fiables. Pour ce verre, la densité moyenne est égale à $2,9071 \mathrm{~g} / \mathrm{cm}^{3}$.

\subsection{Analyse optique}

Le spectre d'absorption de verre dopé erbium a été enregistré à l'aide d'un spectrophotomètre double faisceau l'UV-Vis- Near IR CARY 5G de marque Varian opérant entre 200 et $3000 \mathrm{~nm}$.

\section{Théorie de Judd-Ofelt}

La théorie développée par Judd [4] et Ofelt [5] permet d'obtenir des paramètres $k$ (avec $k=2,4$ et 6 ) qui sont directement reliés à l'intensité des bandes d'absorption et d'émission. Ces paramètres permettent ainsi de prédire l'intensité des pics d'un spectre et donnent des informations sur la covalence entre les ions qui forment un verre [6].

Dans cette théorie, la force d'une transition dipolaire électrique entre deux niveaux $\mid \mathbf{J}$ '> et $\mid \mathbf{J}>$ est donnée par la relation:

$$
S_{e d}^{\text {calc }}=e^{2} \sum_{k=2,4,6} \Omega_{k}\left|\left\langle J^{\prime}|| U^{(k)}|| J\right\rangle\right|^{2}
$$

Les $\quad k$ sont connus sous le nom de paramètres de Judd-Ofelt. Les $U^{(k)}$ sont les composantes de l'opérateur tensoriel réduit reflétant l'approximation du couplage intermédiaire. Les valeurs de $U^{(k)}$ sont constantes et indépendantes de la matrice hôte, et elles sont tabulées [7].

La force de transition dipolaire magnétique $S_{m d}$ expérimentale est définie par la relation suivante $[8]$ :

$$
S_{m d}=\left(\frac{h^{2}}{4 \pi m c}\right)\left|\left\langle J\|L+2 S\| J^{\prime}\right\rangle\right|^{2}
$$

avec $\left|\left\langle J\|L+2 S\| J^{\prime}\right\rangle\right|:$ élément de matrice réduit de l'opérateur de l'élément $\vec{L}+2 \vec{S}$ qui ne dépend que de l'ion terre rare et de la transition $|J\rangle \rightarrow \mid J '>$ considérée [7].

Les forces d'oscillateur sont définies par [9]: 


$$
f\left(J, J^{\prime}\right)=\frac{8 \pi^{2} m c}{3 h(2 J+1) n^{2} \lambda}\left[\chi_{e d} S_{e d}\left(J, J^{\prime}\right)+\chi_{m d} S_{m d}\left(J, J^{\prime}\right)\right]
$$

où: $\chi_{e d}=\frac{n\left(n^{2}+2\right)^{2}}{9}$ et $\chi_{m d}=n^{3}$ sont des termes correctifs du champ local autour de l'ion terre rare, $m$ est la masse de l'électron, $c$ est la célérité de la lumière dans le vide, $n$ est l'indice de réfraction du milieu, $h$ est la constante de Planck et $\lambda$ est la longueur d'onde moyenne de la transition.

Les valeurs des $k$ sont déterminées empiriquement soit à partir des forces de transitions, soit à partir des forces d'oscillateur, en comparant les valeurs calculées à partir de la formule (3) avec les valeurs déterminées expérimentalement à partir des spectres d'absorption à température ambiante. L'intensité d'une bonde d'absorption est proportionnelle avec la force d'oscillateur, expérimentalement, elle donnée par la surface sous la courbe de la bande d'absorption. La force d'oscillateur $f_{\text {mes }}$ peut être exprimée en fonction du coefficient d'absorption $\alpha(\lambda)$ à une longueur d'onde particulière $\lambda$ par la relation [10]:

$$
f_{m e s}=\frac{m c^{2}}{\pi e^{2} N} \int \frac{\alpha(\lambda) d \lambda}{\lambda^{2}}
$$

et comme:

$$
f_{\text {mes }}=f_{e d}{ }^{m e s}+f_{m d}{ }^{m e s}
$$

on a donc:

$$
f_{e d}^{m e s}\left(J, J^{\prime}\right)=\frac{m c^{2}}{\pi e^{2} N} \int_{J \rightarrow J^{\prime}} \frac{\alpha(\lambda) d \lambda}{\lambda^{2}}-f_{m d}^{m e s}
$$

et:

$$
S_{e d}^{m e s}\left(J, J^{\prime}\right)=\frac{9 n}{\left(n^{2}+2\right)^{2}}\left[\frac{3 h c(2 J+1)}{8 \pi^{3} e^{2} N} \int_{J \rightarrow J^{\prime}} \frac{\alpha(\lambda) d \lambda}{\lambda^{2}}-S_{m d}^{m e s}\right]
$$

Soit $q$ le nombre des bandes d'absorption considérées expérimentalement, la résolution d'un système de $q$ équation à 3 inconnues, par une approximation aux moindres carrés, permet d'atteindre les valeurs des paramètres $\quad k$. Chaque transition observée donne lieu l'équation [8]: 


$$
[S]=[U] \cdot[\Omega] \Rightarrow[\Omega]=\left([U]^{T} \cdot[U]\right)^{-1} \cdot[U]^{T} \cdot[S]
$$

Ces paramètres s'expriment en $\mathrm{cm}^{2}$.

Les valeurs des paramètres de Judd-Ofelt obtenus sont utilisées pour calculer les forces des transitions entre l'état initial $J$ et l'état final $J$ '.

\section{RESULTATS ET DISCUSSIONS}

Le spectre d'absorption verre $90 \mathrm{NaPO}_{3}-9 \mathrm{PbCl}_{2}-1 \mathrm{ErF}_{3}$, représenté dans la figure 1 , montre qu'il y a 11 bandes d'absorption résultantes des transitions entre l'état initial ${ }^{4} \mathrm{I}_{15 / 2}$ et les différents états énergétiques de l'ion $\mathrm{Er}^{+3}$.

Les forces de transition mesurés et calculés correspondantes à chaque transition sont illustrées dans le tableau 2. Le facteur de qualité d'ajustement (RMS) est trouvé de l'ordre de $0.8 \times 10^{-20}$, une valeur qui accorde avec les littératures [11-12-13-14-15-16].

Les valeurs des paramètres de Judd-Ofelt obtenus pour notre verre, ainsi les valeurs de le facteur de la qualité spectroscopique $\Omega_{4} / \Omega_{6}$, sont tabulés dans le tableau 3 avec d'autres valeurs de littératures à titre de comparaison. Ces valeurs sont à l'ordre $\Omega_{4}<\Omega_{2}<\Omega_{6}$.

On voit que la valeur de $\Omega_{2}$ du verre $90 \mathrm{NaPO}_{3}-9 \mathrm{PbCl}_{2}-1 \mathrm{ErF}_{3}$ est petite par rapport d'autres verres. Les valeurs de $\Omega_{4}$ et $\Omega_{6}$ sont petits par rapport à la plus part des autres verres.

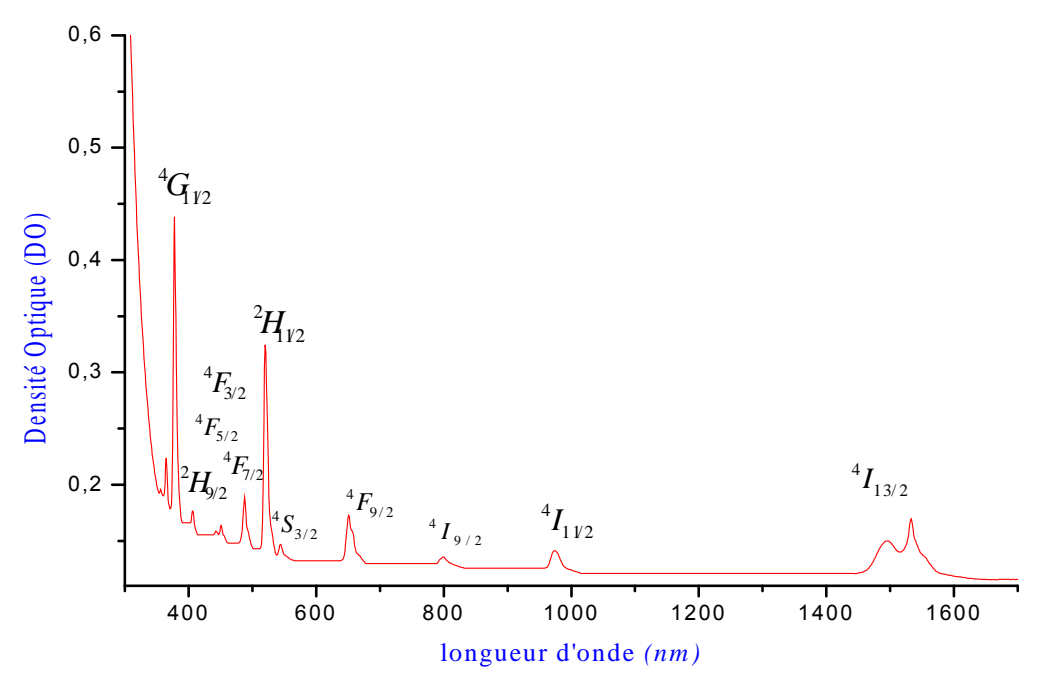

Fig.1. Spectre d'absorption de verre $90 \mathrm{NaPO}_{3}-9 \mathrm{PbCl}_{2}-1 \mathrm{ErF}_{3}$ 
Tableau 2. Forces de transitions $\left(\times 10^{-20} \mathrm{~cm}^{2}\right)$

\begin{tabular}{|c|c|c|}
\hline Transitions $^{4} I_{15 / 2} \rightarrow$ & Expérimental & Calculés \\
\hline${ }^{4} I_{13 / 2}$ & 4.317 & 3.190 \\
\hline${ }^{4} I_{11 / 2}$ & 1.486 & 1.388 \\
\hline${ }^{4} I_{9 / 2}$ & 1.814 & 0.132 \\
\hline${ }^{4} F_{9 / 2}$ & 2.440 & 2.717 \\
\hline${ }^{4} S_{3 / 2}$ & 1.336 & 1.351 \\
\hline${ }^{3} H_{11 / 2}$ & 2.765 & 2.455 \\
\hline${ }^{4} F_{7 / 2}$ & 3.702 & 4.456 \\
\hline${ }^{4} F_{5 / 2}$ & 1.200 & 1.660 \\
\hline${ }^{4} F_{3 / 2}$ & 1.420 & 0.970 \\
\hline${ }^{2} H_{9 / 2}$ & 2.169 & 1.887 \\
\hline${ }^{4} G_{11 / 2}$ & 4.170 & 4.351 \\
\hline Qualité d'ajustement RMS $\left(\times 10^{-20}\right)$ & \multicolumn{2}{|c|}{0.81989} \\
\hline & & \\
\hline & & \\
\hline & & \\
\hline & & \\
\hline
\end{tabular}

Tableau. 3. les paramètres de Judd-Ofelt $\Omega_{2,4,6}\left(\times 10^{-20} \mathrm{~cm}^{2}\right)$

\begin{tabular}{|c|c|c|c|c|c|}
\hline Verres & $\Omega_{2}$ & $\Omega_{4}$ & $\Omega_{6}$ & $\Omega_{4} / \Omega_{6}$ & Ref. \\
\hline Travail actuel & 0.50 & 0.19 & 1.50 & 0,127 & - \\
\hline ZBLAN(fluoré) & 2.20 & 1.40 & 0.91 & 1,538 & $(11)$ \\
\hline Er:ZBLALiP (fluoré ) & 3.12 & 1.49 & 1.15 & 1,296 & $(12)$ \\
\hline NPP0 (chlorophosphate) & 3.36 & 0.51 & 1.51 & 0,338 & $(13)$ \\
\hline NPP30(chlorophosphate) & 3.79 & 0.13 & 1.21 & 0,107 & $(13)$ \\
\hline Chlorophosphate & 1.013 & 0.938 & 0.93 & 1,002 & $(14)$ \\
\hline Fluoroindat & 2.45 & 1.47 & 1.22 & 1.205 & $(15)$ \\
\hline Fluorophosphate & 2.91 & 1.63 & 1.26 & 1,294 & $(16)$ \\
\hline
\end{tabular}


À partir des valeurs de $\Omega_{2}$ et $\Omega_{6}$ obtenus, on peut essayer d'extraire des informations sur les interactions locales des ions $\mathrm{Er}^{-3}$ avec l'environnement du réseau vitreuse comme une fonction de la composition de verre. Le paramètre $\Omega_{2}$ mesure la dégrée de covalence de liaisons chimiques entre $\mathrm{l}^{\prime} \mathrm{Er}^{+3}$ et les atomes proches voisins. La valeur faible de $\Omega_{2}$ indique que les liaisons chimiques liées avec les ions de terres rares dans notre verre est plus ionique que celles des autres verres. Le paramètre $\Omega_{6}$ augment avec la diminution de la rigidité du milieu. La valeur de facteur de la qualité spectroscopique $Q=\Omega_{4} / \Omega_{6}$ égale à 0,127 . Cette valeur est intermédiaire entre celles trouvées pour les verres fluorés et les verres chlorophosphates.

\section{CONCLUSION}

La détermination des paramètres spectroscopiques du verre $90 \mathrm{NaPO} 3-9 \mathrm{PbCl}_{2}-$ $1 \mathrm{ErF}_{3}$, qui passe par l'analyse du spectre d'absorption, montre une fortes Liaisons ioniques entre les ions de $\mathrm{l}^{\prime} \mathrm{Er}^{-3}$ et les plus proches voisins. Elle montre ainsi une rigidité de ce verre par rapport aux autres verres.

Les résultats obtenus peuvent être utilisé, prochainement, pour déterminer des autres paramètres spectroscopiques, comme la durée de vie et section d'émission, après avoir le spectre d'émission de notre verre.

\section{REFERENCES}

[1] Binnemans K., Deun R.V., Görller-Walrand C., Adam J. L. Spectroscopic properties of trivalent lanthanide ions in fluorophosphate glasses. J. Non-Cryst. Solids. 1998, 11, 238.

[2] Zhang G.Y., Poulain M. J. Influence of rare-earth ions on fluorogallate glass formation and properties. J. Alloys Compd. 1998, 15, 275-277.

[3] Philipps J. F., Töpfer T., Ebendorff-Heidepriem H., Ehrt D., Sauerbrey R. Spectroscopic and lasing properties of $\mathrm{Er}^{3+}: \mathrm{Yb}^{3+}$-doped fluoride phosphate glasses. Appl. Phys. 2001, B 72, 399.

[4] Judd B. R. Phys. Rev. 1962, 127, 750.

[5] Ofelt G. S. J. Chem. Phys. 1962, 37, 511. 
[6] Reisfeld R. and Jorgensen C. K. 1987, In Handbook on the Physics and Chemistry of Rare Earth, Edited by K. A. Gschneider and L. Eyring, North-Holland, Amesterdam, Chap 58.

[7] Carnnall W.T., Fields P. R. and Wybourne B. G. Spectral intensities of the trivalente Lanthanides and Actinides in solution.I. The Journal of Chemecal Physics. 1965, 42(11).

[8] Petit L. (2002), Amplification optique dans des verres borophosphate de niobium et tellurite dopés aux ions de terres rares présentant un indice optique non linéaire élevé, Thèse Doctorat, Université Bordeaux 1.

[9] Reisfeld R. Rare earth complexes in sol-gel glasses. Materials Sciences. 2002, 20(2). [10] Saisudha M. B. and Ramakrishna J. Effect of host glass on the optical absorption properties of $\mathrm{Nd}^{3+}, \mathrm{Sm}^{3+}$, and $\mathrm{Dy}^{3+}$ in lead borate glasses. Physical Review B. 1996, 53(10).

[11] McDougall J., Hollios D. B. and Payne M. J. Spectroscopic properties of $\mathrm{Er}^{3+}$ in ZBLAN fluoride glass. Phys.Chem. Glasses. 1996, 37, 256.

[12] Mortier M., Goldner P., Feron P., Stephan G. M., Xu H., Cai Z. New fluoride glasses for laser applications. Journal of Non-Crystalline Solids 326\&327. 2003, 505509.

[13] Pradeesh K., Oton C. J., Agotiya V. K., Raghavendra M., Vijaya Prakash G. Optical properties of $\mathrm{Er}^{3+}$ doped alkali chlorophosphate glasses for optical amplifiers. Optical Materials. 2008, 31155-160.

[14] Ratnakaram Y. C., Viswanadha Reddy A., Sreekanth Chakradhar R. P. Electronic absorption spectra and energy gap studies of $\mathrm{Er}^{3+}$ ions in different chlorophosphate glasses. Spectrochimica Acta Part A. 2002, 58, 1809-1822.

[15] Florez A., Messaddeq Y., Malta O. L., Aegerter M. A. Optical transition

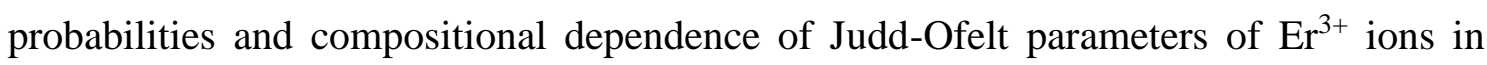
fluoroindate glass. Journal of Alloys and Compounds. 1995, 227, 135-140.

[16] Yamauchi H., Ohishi Y. Spectroscopic properties of $\mathrm{Er}^{3+}$-doped $\mathrm{PbO}-\mathrm{Ga}_{2} \mathrm{O}_{3}-\mathrm{GeO}_{2}$ glass for optical amplifiers. Optical Materials. 2005, 27, 679-690. 


\section{APPLICATION DE LA THÉORIE DE JUDD-OFELT SUR UN VERRE CHLOFLUOROPHOSPHATE DOPÉ ERBIUM}

\section{RESUME}

Une séries de verres chlorofluorophosphates dopés Erbium ont été préparés et caractérisés. Les spectres d'absorption ont été analysés pour déterminer les paramètres de Judd-Ofelt. Les performances optiques de ces verres dopés suggérant la pertinence de ces verres pour les fibres optiques / guides d'ondes lasers et amplificateurs optiques.

Mots-clés: Verre dopé, terres rares, erbium, Paramètres de Judd-Ofelt, laser, amplificateur optique.

\section{How to cite this article}

Babai A, Aiadi K E, Bentouila O, Rehoma F and Poulain M. Application of Judd-Ofelt theory upon chlofluorophosphate glass doped with erbium. J Fundam Appl Sci. 2012, 4(1), 64-72. 